\title{
A PASSIVE MICROMECHANICAL FREQUENCY DIVIDER
}

\author{
B.S. Strachan ${ }^{1,2}$, K.R. Qalandar ${ }^{3}$, B. Gibson ${ }^{3}$, M. Sharma ${ }^{3}$, P. Polunin ${ }^{1,4}$, S.W. Shaw ${ }^{1}$, and K.L. Turner ${ }^{3}$ \\ ${ }^{1}$ Department of Mechanical Engineering, Michigan State University, East Lansing, MI USA 48824 \\ ${ }^{2}$ Department of Electrical Engineering, Michigan State University, East Lansing, MI USA 48824 \\ ${ }^{3}$ Department of Mechanical Engineering, University of California, Santa Barbara, CA, USA 93106 \\ ${ }^{4}$ Department of Physics and Astronomy, Michigan State University, East Lansing, MI USA 48824
}

\begin{abstract}
We report on the design and experimental characterization of a passive MEMS frequency divider that is based on a cascade of parametrically coupled nonlinear resonator vibration modes. The device is designed to have 1:2 frequency ratios between adjacent modes with an orientation that promotes the necessary nonlinear modal coupling. We characterize the fabricated device and map the normalized parametric instability parameter regions for the first two modes. We successfully activate the cascade by parametrically driving the second mode and, by operating in the intersection of the instability regions, the energy cascades to the first mode, resulting in an input-to-output frequency ratio of four.
\end{abstract}

\section{INTRODUCTION}

Passive frequency conversion is an essential component for sensor networks and is ideal for critical applications requiring low power and good phase noise characteristics, such as in frequency synthesizers[1]. Currently, passive frequency dividers for RF applications are limited to electronic topologies which are large compared to MEMS resonators[2]. The divider device presented here is based on a cascade of subharmonic resonances in a set of $N$ vibration modes tuned with relative $1: 2$ frequency ratios [3]. It takes an input signal of $423.6 \mathrm{kHz}$ and passively divides it through two modal stages, producing signals at $211.8 \mathrm{kHz}$ and $105.9 \mathrm{kHz}$. We specify the operating region for this system in the drive frequency and drive amplitude parameter space. This novel divider design has simple design rules and can be extended to a large number of modal stages, ultimately dividing by $2^{N}$.

\section{FREQUENCY DIVIDER}

Figure 1 shows an SEM image of the fabricated device along with a diagram of the conceptual scheme on which it is based [3]. It consists of a set of flexible beams that are orthogonally coupled, providing parametric driving from the shorter beam to the adjacent longer beam. The released structure is fabricated in single-crystal silicon. The microbeam elements are uniformly $10 \mu \mathrm{m}$ in depth and $1.85 \mu \mathrm{m}$ wide, and the beam lengths increase by approximately $\sqrt{2}$ to tune adjacent modal frequencies to the required $1: 2$ ratios. The device is driven capacitively, providing a force proportional to the DC and AC applied voltages. The velocity of the in-plane motion of each beam is detected using a Polytec UHF-120 Laser Doppler Vibrometer. To direct the laser beam perpendicular to the motion, $45^{\circ}$ angled mirrors are etched using focused ion beam (FIB) milling. Measurements are performed at a driving voltage of $100 V_{D C}$ and $0-50 V_{A C}$ at a pressure of $\sim 400$ mTorr.

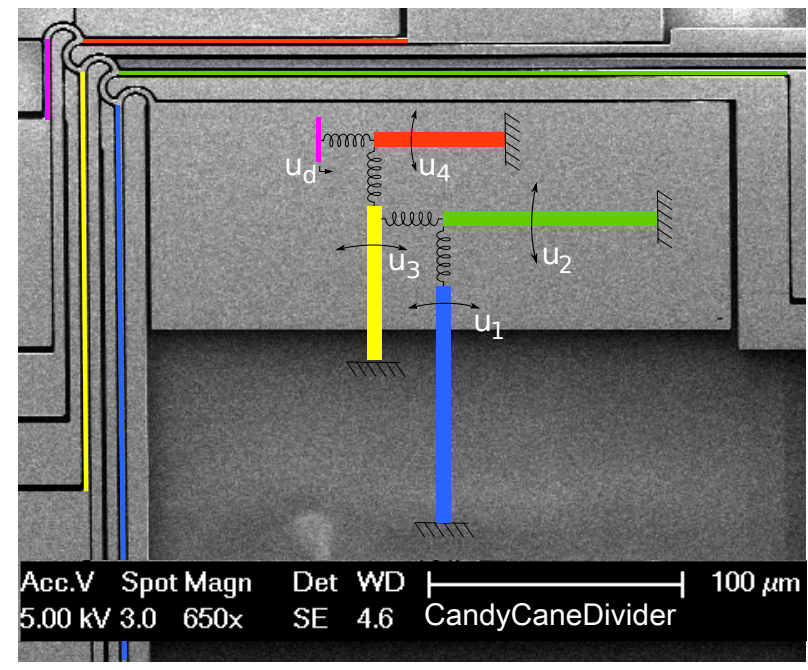

Figure 1: An SEM image of the fabricated device; inset shows the conceptual model that inspired the design [3].

For this device, each mode is spatially localized in a given beam in order to promote the required nonlinear intermode coupling. The high frequency mode (beam) is driven parametrically at twice its natural frequency. This beam, in the desired operating regime, will parametrically drive the next beam, and so on down the cascade, due to the $1: 2$ frequency ratios, their relative orientation, and nonlinear modal coupling. The device of Figure 1 has four beams; we were able to activate only the first and second modes, resulting in division by four, due to modal frequency mistuning. Figure 2 shows the first two vibration modes for a COMSOL model of the device, with the required $1: 2$ frequency ratio. Simulations of this COMSOL model in fact show division by 16 , and this should be achievable in devices with more precise modal tuning.

\section{RESULTS}

Figure 3 shows the regions of drive amplitude and frequency for which modes 1 and 2 are individually excited into parametric resonance. The overlap region is where the cascade is expected to become activated. Figure 4 shows the measured response amplitudes from modes 1 and 2, and theoretical response curves from [3], over the frequency range of interest. Note that the mode 2 amplitude drops when mode 1 becomes active. The presence of a mode 1 response represents passive frequency division by a factor of 4 . 


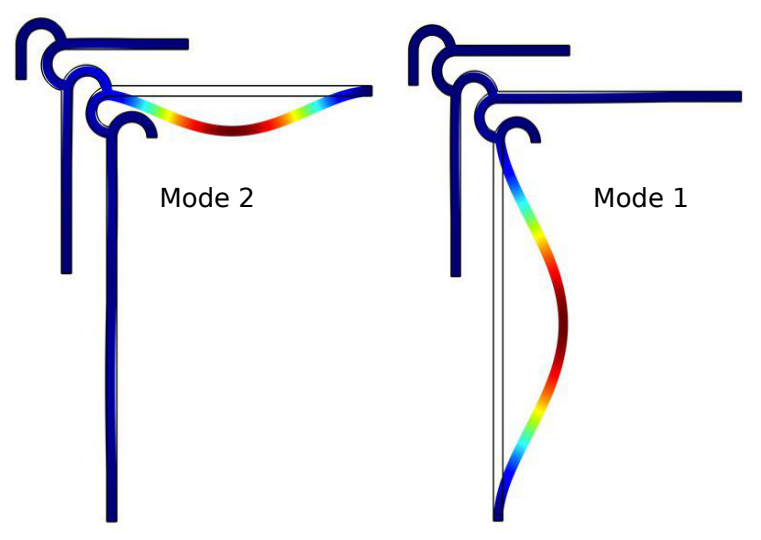

Figure 2: First two vibration modes from a COMSOL model. Mode frequencies are $105.95 \mathrm{kHz}$ (mode 1) and $211.7 \mathrm{kHz}$ (mode 2).

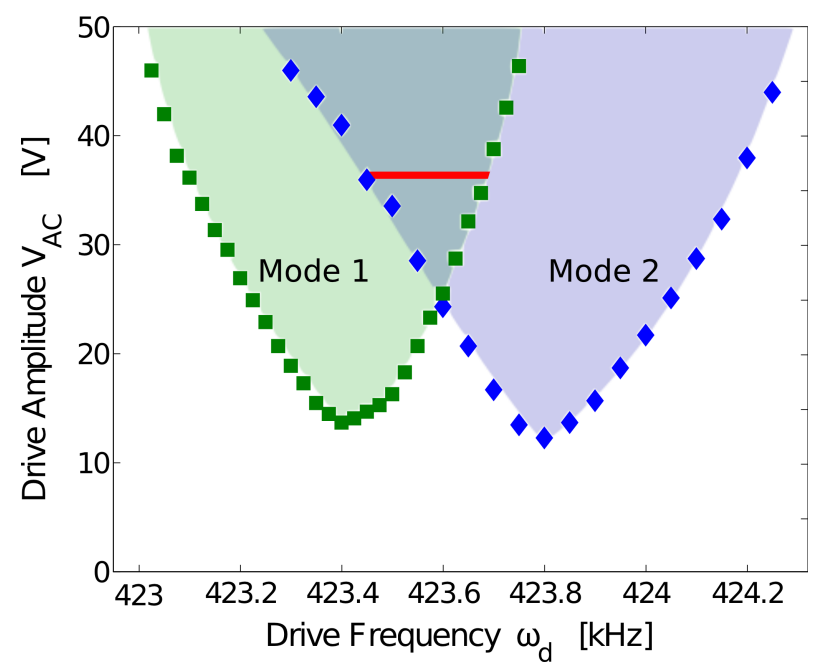

Figure 3: Parametric resonance zones for the first two modes of the device. The shaded overlap region is where the divideby-four operation is achieved. The red segment indicates the operating voltage and frequency range of interest.

\section{CONCLUSION}

The results demonstrate passive frequency division in MEMS using a cascade of parametric resonances. Division by $2^{N}$ requires mutual overlap of $N$ regions such as those shown in Fig. 3, which can be achieved for larger $N$ by improved tuning of device frequencies, a topic of current investigation. Also of interest are methods for designing devices with desired levels of nonlinear modal coupling, which will promote activation of the cascade, and the phase noise performance of these devices.

\section{ACKNOWLEDGMENTS}

We thank Alice Ma for her preliminary work on the divider design. This work was supported by an NSF collaborative project under grants 1234067 and 1234645.

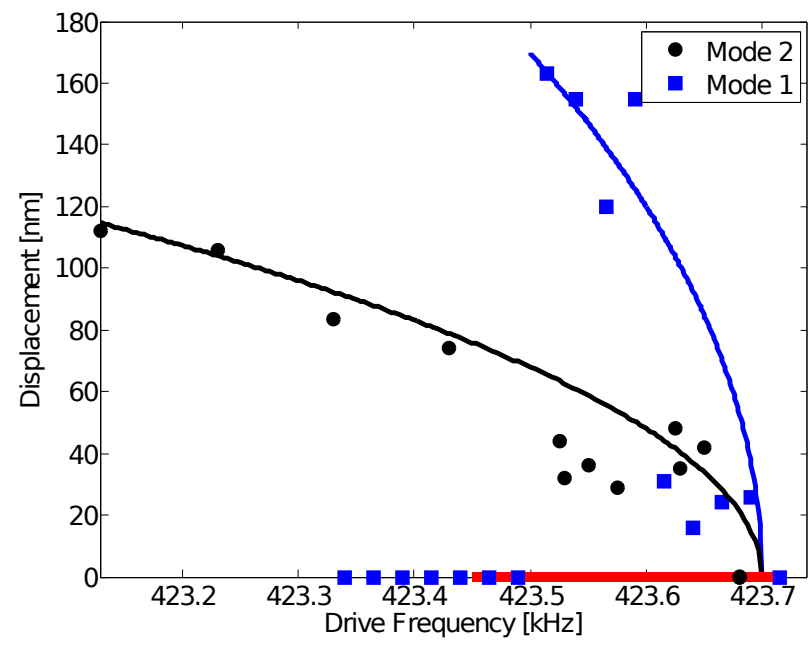

Figure 4: Measured mode 1 and 2 amplitudes, extracted from the spectrum at $1 / 2$ and $1 / 4$ of the drive frequency from the response measured at the center of the respective mode localized beam, versus drive frequency, along with curve fits based on the analytical model [3], over the frequency range of interest. The AC driving amplitude is $36 \mathrm{~V}$. The frequency range marked with red corresponds to that of Figure 3, where the cascade is predicted to occur. Note that these data were taken on separate sweeps, since simultaneous measurement of both modes was not possible, and that the mode 1 response data was slightly frequency shifted to account for drift between the runs.

\section{REFERENCES}

[1] M.M. Driscoll, "Phase Noise Performance of Analog Frequency Dividers", IEEE Transactions on Ultrasonics, Ferroelectrics and Frequency Control, 37, 4 (1990).

[2] J. Lee, B. Razavi,"A 40-Ghz Frequency Divider in 0.18mm CMOS Technology", IEEE Journal of Solid State Circuits, 39, 4 (2004).

[3] B.S. Strachan, S.W. Shaw, and O. Kogan, "Subharmonic Resonance Cascades in a Class of Coupled Resonators", Journal of Computational and Nonlinear Dynamics, 8, 4 (2013). 